University of Wollongong

Research Online

Faculty of Engineering and Information

Faculty of Engineering and Information

Sciences - Papers: Part A

Sciences

$1-1-2013$

\title{
Three-dimensional measurement of object surfaces with complex shape and color distribution based on projection of color fringe patterns
}

\author{
Ke Chen \\ University of Wollongong, kc245@uowmail.edu.au \\ Jiangtao Xi \\ University of Wollongong, jiangtao@uow.edu.au \\ Yanguang Yu \\ University of Wollongong, yanguang@uow.edu.au \\ Sheng Tong \\ University of Wollongong, sheng@uow.edu.au \\ Qinghua Guo \\ University of Wollongong, qguo@uow.edu.au
}

Follow this and additional works at: https://ro.uow.edu.au/eispapers

Part of the Engineering Commons, and the Science and Technology Studies Commons 


\title{
Three-dimensional measurement of object surfaces with complex shape and color distribution based on projection of color fringe patterns
}

\begin{abstract}
A challenging issue associated with three-dimensional (3D) fringe patterns profilometry (FPP) is the unwrapping of phase maps resulting from color object surfaces. This paper proposes a new colorprojection-based 3D FPP, making use of the three primary color channels [i.e., red, green, and blue (RGB)] associated with digital projectors. One channel (e.g., red) is used for projecting sinusoidal fringes required by phase shift profilometry (PSP); the other two channels are employed for generating binary stripe patterns. In order to achieve reliable phase unwrapping, each fringe of the sinusoidal patterns is identified by a unique binary sequence. These sequences are then encoded by a channel-encoding scheme used in the area of communication. The encoded sequences are embedded in the binary coding stripe images, which are projected together with the sinusoidal patterns. The three image patterns are reflected by the object surface and captured by an RGB 3-CCD camera. The reflected sinusoidal patterns are employed to yield a wrapped phase map, and the binary stripe patterns are used to retrieve the encoded sequences, which are then decoded to yield the original binary sequences for phase unwrapping. Compared with existing color-encoded algorithms, the proposed approach uses binary codes instead of fringe color to identify the fringes, which are less sensitive to the disturbances caused by object surface color and illumination noises. Furthermore, use of the channel-coding scheme provides extra resistance to the disturbances caused by object surface color and illumination noises. Experimental results are presented to confirm the effectiveness of the proposed technique.
\end{abstract}

\section{Keywords}

shape, complex, surfaces, object, measurement, patterns, dimensional, fringe, three, projection, distribution, color

\section{Disciplines}

Engineering | Science and Technology Studies

\section{Publication Details}

K. Chen, J. Xi, Y. Yu, S. Tong \& Q. Guo, "Three-dimensional measurement of object surfaces with complex shape and color distribution based on projection of color fringe patterns," Applied Optics, vol. 52, (30) pp. 7360-7366, 2013. 


\title{
Three-dimensional measurement of object surfaces with complex shape and color distribution based on projection of color fringe patterns
}

\author{
Ke Chen, Jiangtao Xi, ${ }^{*}$ Yanguang Yu, Sheng Tong, and Qinghua Guo \\ School of Electrical, Computer \& Telecommunications Engineering, University of Wollongong, \\ Keiraville 2500, NSW, Australia \\ *Corresponding author: jiangtao@uow.edu.au
}

Received 29 July 2013; revised 25 September 2013; accepted 1 October 2013; posted 2 October 2013 (Doc. ID 194791); published 18 October 2013

\begin{abstract}
A challenging issue associated with three-dimensional (3D) fringe patterns profilometry (FPP) is the unwrapping of phase maps resulting from color object surfaces. This paper proposes a new colorprojection-based 3D FPP, making use of the three primary color channels [i.e., red, green, and blue (RGB)] associated with digital projectors. One channel (e.g., red) is used for projecting sinusoidal fringes required by phase shift profilometry (PSP); the other two channels are employed for generating binary stripe patterns. In order to achieve reliable phase unwrapping, each fringe of the sinusoidal patterns is identified by a unique binary sequence. These sequences are then encoded by a channel-encoding scheme used in the area of communication. The encoded sequences are embedded in the binary coding stripe images, which are projected together with the sinusoidal patterns. The three image patterns are reflected by the object surface and captured by an RGB 3-CCD camera. The reflected sinusoidal patterns are employed to yield a wrapped phase map, and the binary stripe patterns are used to retrieve the encoded sequences, which are then decoded to yield the original binary sequences for phase unwrapping. Compared with existing color-encoded algorithms, the proposed approach uses binary codes instead of fringe color to identify the fringes, which are less sensitive to the disturbances caused by object surface color and illumination noises. Furthermore, use of the channel-coding scheme provides extra resistance to the disturbances caused by object surface color and illumination noises. Experimental results are presented to confirm the effectiveness of the proposed technique. (C) 2013 Optical Society of America

OCIS codes: (110.5086) Phase unwrapping; (120.2650) Fringe analysis; (120.5050) Phase measurement; (120.6650) Surface measurements, figure.

http://dx.doi.org/10.1364/AO.52.007360
\end{abstract}

\section{Introduction}

Fringe patterns profilometry (FPP) has been an active area of research as an effective tool for noncontact three-dimensional (3D) shape measurement, with many applications, such as automatic manufacturing, machine vision, medical services, and antiques protection, etc. [1-6]. A typical FPP system consists of a digital projector, a CCD camera, and a computer. With FPP, a group of sinusoidal light

$1559-128 \mathrm{X} / 13 / 307360-07 \$ 15.00 / 0$

(C) 2013 Optical Society of America patterns is projected onto the object surface, which are deformed by the object surface shape. As the deformation can be considered as the result of phase modulation of the projected patterns, detection of the phase shift yields the shape of the object surface. During the recent two decades, various methods have been proposed to retrieve the phase from reflected fringe patterns, such as Fourier transform profilometry (FTP) and phase-shifting profilometry (PSP). However, the phase values retrieved by FTP and PSP are wrapped into the range from $-\pi$ to $\pi$, resulting in discontinuities on the phase map. In order to obtain the $3 \mathrm{D}$ shapes, absolute phases are 
required, which must be recovered from the wrapped ones. This process is known as phase unwrapping, which is a critical step in the implementation of FPP.

Although phase unwrapping is quite simple in principle, its implementation is a challenging task. If the true phase is slow varying, phase unwrapping can be implemented by adding or subtracting $2 \pi$ to make the difference of relative phase values of neighboring pixels lies in the range $\pi$. However, when large depth discontinuities are observed, the wrong decision of unwrapping may be made regarding the addition or subtraction of $2 \pi$. In order to implement reliable and accurate unwrapping, intensive studies have been devoted, and various approaches have been developed, including the quality-guided methods [2-5], the temporal method [6-10], and color-projectionbased methods [11-18]. With quality-guided methods, every pixel on the phase maps is associated with a quality value measuring the smoothness of the pixel in contrast to its surrounding ones. The unwrapping process starts from the pixel with the highest quality and proceeds to the lower-quality ones until all pixels are unwrapped. The unwrapping path can change its direction in order to avoid the large depth discontinuities. However, when an object surface observes isolated areas, such an approach may not work. Another class of phase-unwrapping approaches is temporal techniques. With the temporal techniques, a set of fringe patterns with different spatial frequencies are projected; each yields a wrapped phase map. Phase unwrapping is completed by comparing those phase maps. While the temporal method is able to recover phase maps, a large number of fringe patterns are required in the cases of large depth discontinuities on the object surface.

With the development of digital-color-projection systems and 3-CCD cameras, the FPP technique is no longer restricted to monochrome fringe patterns. Several color-projection-based approaches are proposed [11-16]. Wang et al. [11] proposes a colorembedded FTP, where every individual fringe is associated with a particular color, and phase unwrapping can be implemented by retrieving the color of each fringe. However, when a large number of fringes are projected, the number of colors must also be large. This will result in a reduction in the difference among the colors, making them difficult to be identified. The problem becomes worse when the fringes are corrupted by object surface color. Su [12] proposes a color-encoded FTP. In this method, each sinusoidal fringe is identified by three different color stripes, where each color stripe covers $1 / 3$ width of a sinusoidal fringe. Using such an approach, large fringe numbers will not reduce the difference of color as many different combinations of color stripes can be used. However, the color stripes may be missed at large depth discontinuities, resulting in a phaseunwrapping error. Chen et al. [14] proposes a color-projection-based structure light system in which the fringes are binary stripes, which are identified by different colors. 3D data can be retrieved by recording the deformation of the width of binary stripes. This method does not contain a phasedetection scheme and thus is faster than the sinusoidal fringe-based methods. However, the measurement precision is related to the width of the binary stripe, which has to be very narrow; thus this method is sensitive to the optical defocusing introduced by the projector and camera.

Existing color-encoding approaches mentioned above all employ a different color to differentiate individual fringes for phase unwrapping. The antierror capability associated with these approaches depends on the difference in the color used for identifying different fringes. The surface color has a significant impact on the difference, which could become rather small, leading to significant degradation of the anti-error capability. In this paper, we propose a new color-projection-based 3D FPP approach where each sinusoidal fringe is identified by a unique binary sequence. These sequences are designed to have the maximal difference using the channel encoding theory in digital communication systems. With the color-projection technique, the sinusoidal fringes and binary stripes are projected simultaneously using red, green, and blue (RGB) colors. Then the reflected images are captured by 3-CCD camera and decomposed into RGB components. The wrapped phase map is obtained from the sinusoidal fringe patterns by means of PSP techniques. The phase unwrapping is implemented based on the binary sequences retrieved from the binary coding stripes. Compared with existing color-projection-based approaches [11-14], this approach uses binary codes instead of fringe color to identify the fringes, which is less sensitive to the disturbances caused by object surface color. Furthermore, the coding scheme used in this approach provides extra resistance to the disturbances. The experiment results show that our approach is able to be applied to the measurement of objects with both surface color distribution and large depth discontinuities.

This paper is organized as follows. In Section 2 , the approach is presented. In Section $\underline{3}$, experiment results are demonstrated to verify the effectiveness of the proposed approach. Finally, the conclusions are drawn in Section 4 .

\section{Description of Algorithm}

\section{A. Basic Principle of PSP}

In $M$-step PSP [2,3], $M$ frames of sinusoidal fringe patterns are projected onto a reference plane and then the object surface. The reflected images on reference plane $s_{m}(x, y)$ and object surface $d_{m}(x, y)$ captured by the CCD camera are as follows:

$$
s_{m}(x, y)=R_{s}\left[I_{1}+I_{2} \cos \left(\phi_{s}(x, y)+\frac{2 \pi(m-1)}{M}\right)\right],
$$


$d_{m}(x, y)=R_{d}(x, y)\left[I_{1}+I_{2} \cos \left(\phi_{d}(x, y)+\frac{2 \pi(m-1)}{M}\right)\right]$,

where $m=1,2, \ldots, M . \phi_{s}(x, y)$ is the phase on reference plane. $\phi_{d}(x, y)$ is the phase on object surface, which contains the object surface 3D information. $R_{s}$ is the reflectivity on reference plane, which is assumed to be constant. $R_{d}(x, y)$ is the reflectivity on the object surface. $I_{1}$ and $I_{2}$ are the illumination intensity and fringe intensity, respectively, which are assumed to be constant. Once the $\phi_{s}(x, y)$ and $\phi_{d}(x, y)$ are obtained, we can calculate the phase shift $\phi(x, y)=\phi_{d}(x, y)-\phi_{s}(x, y)$. Height of the object surface $h(x, y)$ can be acquired by the following:

$$
h(x, y)=\frac{l_{0} \phi(x, y)}{\phi(x, y)-2 \pi f_{0} d_{0}},
$$

where $l_{0}$ is the distance between the camera and the reference plane, and $d_{0}$ is the distance between the camera and the projector. We also assume that each fringe covers $m_{0} \mathrm{~mm}$ on the reference plane, and $f_{0}=1 / m_{0}$ is the fundamental frequency. Hence, a major issue in FPP is to determine $\phi_{s}(x, y)$ and $\phi_{d}(x, y)$ based on the projected fringe patterns. However, instead of the true ones, wrapped versions of them are obtained with their value wrapped into the range $(-\pi, \pi]$. These wrapped phases, denoted by $\phi_{s}^{r}(x, y)$ and $\phi_{d}^{r}(x, y)$, are related to the true ones as follows:

$$
\begin{aligned}
& \phi_{s}^{r}(x, y)=\left[\phi_{s}(x, y)\right]_{\bmod 2 \pi}, \\
& \phi_{d}^{r}(x, y)=\left[\phi_{d}(x, y)\right]_{\bmod 2 \pi},
\end{aligned}
$$

where $[\cdot]_{\bmod 2 \pi}$ denotes the modular operation making the argument to be within $(-\pi, \pi]$. In order to obtain $h(x, y), \phi_{s}(x, y)$ and $\phi_{d}(x, y)$ must be recovered from $\phi_{s}^{r}(x, y)$ and $\phi_{d}^{r}(x, y)$, and such an operation is referred to as phase unwrapping, which is the reverse operations of Eqs. (ㄴ) and (ㅁ), as follows:

$$
\begin{aligned}
& \phi_{s}(x, y)=2 \pi \cdot k_{s}(x, y)+\phi_{s}^{r}(x, y), \\
& \phi_{d}(x, y)=2 \pi \cdot k_{d}(x, y)+\phi_{d}^{r}(x, y) .
\end{aligned}
$$

The two integers $k_{s}(x, y)$ and $k_{d}(x, y)$ are also referred to as fringe number indices, with its value indicating the location of $x$ in the patterns. For example, $k_{d}(x, y)=n-1$ implies that $\phi_{d}(x, y)$ is at the $n$th fringe on the patterns. Phase unwrapping is such a process that aims at retrieving $k_{s}(x, y)$ and $k_{d}(x, y)$ for each pixel. As $\phi_{s}(x, y)$ is a linear function with respect to $x, \phi_{s}^{r}(x, y)$ is also a linear function within a fringe period, which is easy to be unwrapped. Hence we only consider unwrapping of $\phi_{d}^{r}(x, y)$ in the rest of this paper.

\section{B. Composite Fringe Patterns Projection}

As discussed above, phase unwrapping is to retrieve the fringe number index $k_{d}(x, y)$. In this paper, we propose to associate each of the fringes to a digital binary sequence, which is then employed to retrieve $k_{d}(x, y)$. In order to achieve this, we employ the three primary colors (i.e., RGB) to generate three images. One image is sinusoidal fringe patterns for PSP, and the other two are binary stripes, which are used to encode the binary sequences in order to identify $k_{d}(x, y)$ for phase unwrapping.

Let us utilize an example to demonstrate the implementation of the method described above. Without loss of generality, we assume that the sinusoidal patterns are projected in red, and the two binary stripe patterns are in green and blue, respectively. In order to implement an $M$-step PSP, $M$ frames of color images are projected, which is the combination of $M$ sinusoidal fringe patterns in red and $2 M$ binary strip patterns in green and blue. The sinusoidal patterns are equally shifted in their initial phase as following:

$$
g_{m}(x, y)=I_{1}+I_{2} \cos \left[2 \pi f_{0} x+\frac{2 \pi(m-1)}{M}\right],
$$

where $m=1,2, \ldots, M \cdot g_{m}(x, y)$ is a periodic function of $x$, each period corresponding to a fringe. Assuming there are $N$ fringes on $g_{m}(x, y), N$ different binary sequences $q_{n}(n=1,2, \ldots, N)$ are required to identify the fringe number index $k_{d}(x, y)$, implying that the length of each $q_{n}$ must be longer than $\left\lceil\log _{2} N\right\rceil$ bits.

As mentioned above, the $2 M$ binary stripe patterns are employed to carry the digital sequences, which are projected together with the sinusoidal patterns with the aim to identify the fringe number indices. However, the binary stripes are often disturbed by object surface color or environmental illumination. If we consider the binary stripes as signals, such disturbances can be modeled as interferences and noises to the signal. To cope with disturbances, we can employ the channel-coding technique [18-20], which encodes a length $k$ message sequence by adding $(n-k)$ redundant bits in a controlled manner to produce a length $n$ bit sequence, called a codeword, and thus yields extra resistance to such interferences and noises. The set of all codewords is called an $(n, k)$ code. In the proposed approach, we encode each $q_{n}$ with length $k$ into a length $2 M$ codeword $C_{n}$. The codeword $C_{n}$ is then transmitted as binary stripes, which will be corrupted by the disturbances. When implementing phase unwrapping at the receiver side, this corrupted binary stripe is then decoded according to the structure introduced by the channel code, and an estimation of $q_{n}$ is generated. It is well known that the correction capability of a channel code, i.e., the number of error bits the code can correct, is determined by its minimum Hamming distance $d_{H}$ between codewords. A channel code with a minimum Hamming distance of $d_{H}$ can correct $\left\lfloor\left(d_{H}-1\right) / 2\right\rfloor$ bit errors [18]. Consider an example 


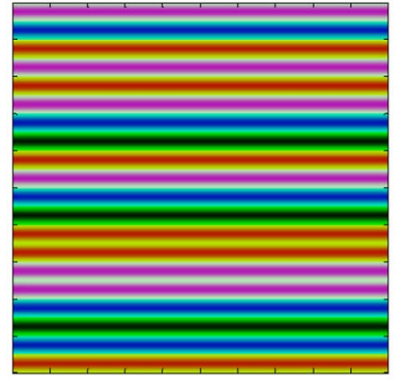

(a)

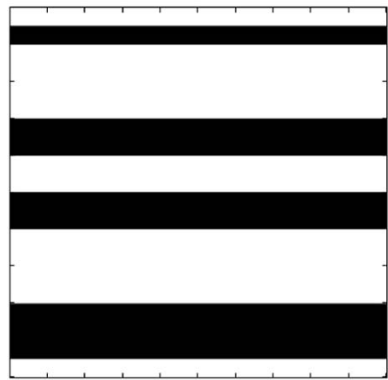

(c)

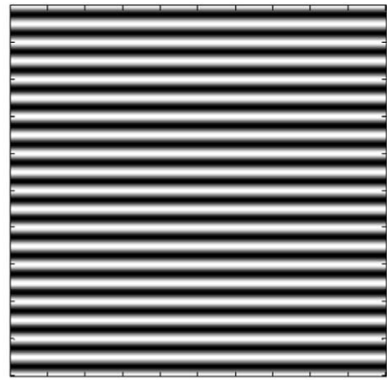

(b)

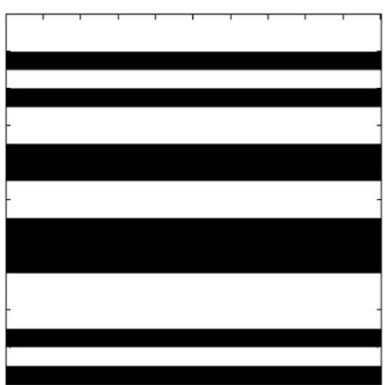

(d)
Fig. 1. (a) Color-coded fringe patterns at $m$ th frame. The order of fringes is from top to bottom. (b) Sinusoidal fringes in red. (c) Binary stripes in green, $N=20$. (d) Binary stripes in blue, $N=20$.

where the number of fringe patterns is 20 , the shortest message length will be $k=5$. As the length of $C_{n}$ is even, we considered $(6,5),(8,5),(10,5)$ and $(12,5)$ binary linear block codes. The corresponding upper bounds on the minimum Hamming distances are $2,2,4$, and 4 . In consideration of the minimum distance and code length, we attempt to construct a $(10,5)$ code with a minimum distance of 4 . In fact, we can build such a code by fixing the first six message bits of the $(16,11)$ extended Hamming code to be 0 , which leads to a $(10,5)$ shortened extended Hamming code with a minimum distance of 4 .

Once the $C_{n}$ is obtained, the binary stripes in blue and green are generated based on $C_{n}$. If we denote $C_{n}$ as $C_{n}=\left\{a_{1 n}, a_{2 n}, \ldots, a_{M n}, b_{1 n}, b_{2 n}, \ldots, b_{M n}\right\}, \quad$ each element in $C_{n}$ is a binary codeword " 1 " or " 0. " The first half of $C_{n}$, which is $\left\{a_{1 n}, a_{2 n}, \ldots, a_{M n}\right\}$, determines the intensity of the $n$th binary stripe in green from the first frame to the $M$ th frame. The second half of $C_{n}$, which is $\left\{b_{1 n}, b_{2 n}, \ldots, b_{M n}\right\}$, determines the intensity of the nth binary stripe in blue from the first frame to the $M$ th frame. The intensity of binary stripe has two levels: bright or dark, corresponding to " 1 " or " 0 " in $C_{n}$. We use two matrices, $\mathbf{A}$ and $\mathbf{B}$, to denote the intensity of binary stripes to be projected:

$$
\begin{aligned}
\mathbf{A} & =\left(\begin{array}{cccc}
a_{11} & a_{12} & \ldots & a_{1 N} \\
a_{21} & a_{22} & \ldots & a_{2 N} \\
\ldots & & & \\
a_{M 1} & a_{M 2} & \ldots & a_{M N}
\end{array}\right), \\
\mathbf{B} & =\left(\begin{array}{cccc}
b_{11} & b_{12} & \ldots & b_{1 N} \\
b_{21} & b_{22} & \ldots & b_{2 N} \\
\ldots & & & \\
b_{M 1} & b_{M 2} & \ldots & b_{M N}
\end{array}\right),
\end{aligned}
$$

where the transpose of $n$th column in $\mathbf{A}$ is the first half of $C_{n}$, and the transpose of $n$th column in B are the second half of $C_{n}$. Codeword $a_{m n}$ in matrix A determines the intensity of the $n$th binary stripe in green at the $m$ th frame. Similarly, codeword $b_{m n}$

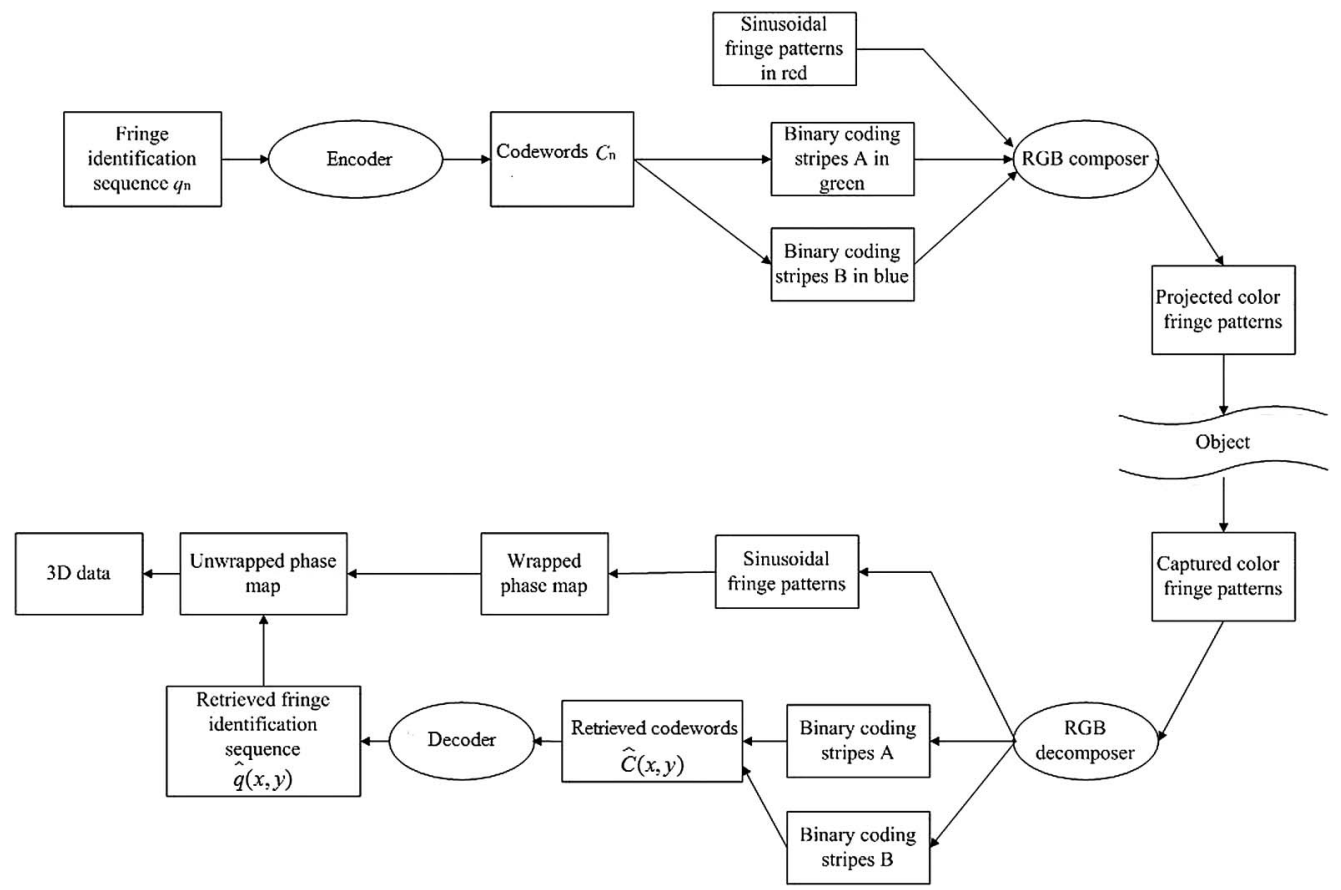

Fig. 2. Flowchart of the proposed approach. 


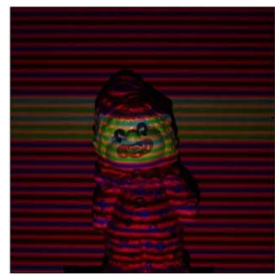

(a)

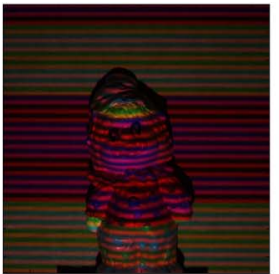

(b)

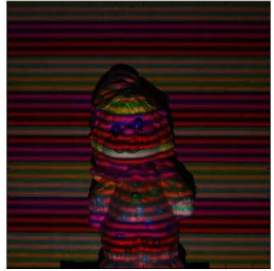

(c)

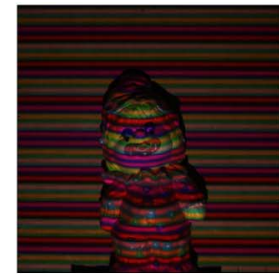

(d)

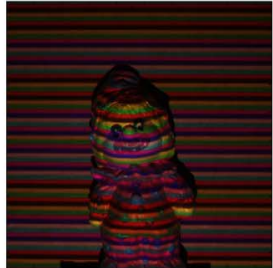

(e)

Fig. 3. Captured images of (a) the first frame, (b) the second frame, (c) the third frame, (d) the fourth frame, and (e) the fifth frame of the composite fringe patterns.

in matrix $\mathbf{B}$ determines the intensity of $n$th binary stripe in blue at the $m$ th frame.

Figure 1 shows an example of the composite fringe patterns at the $m$ th frame and their RGB components, where $N=20$ and $M=5$. The binary stripes in green, which start from the top to the bottom are determined by the codewords $\left\{a_{m 1}, a_{m 2}, \ldots, a_{m 20}\right\}$, which is the $m$ th row of matrix $\mathbf{A}$, where $\left\{a_{m 1}, a_{m 2}, \ldots, a_{m 20}\right\}=\{1,0,1,1,1,1,0,0,1,1,0,0,1,1$, $1,1,0,0,0,1\}$. The binary stripes in blue, which start from the top to the bottom, are determined by codewords $\left\{b_{m 1}, b_{m 2}, \ldots, b_{m 20}\right\}$, where $\left\{b_{m 1}, b_{m 2}, \ldots, b_{m 20}\right\}=$ $\{1,1,0,1,0,1,1,0,0,1,1,0,0,0,1,1,1,0,1,0\}$, which is the $m$ th row of matrix $\mathbf{B}$.

\section{Phase Unwrapping}

Once the composite fringe patterns are captured by the 3-CCD camera, they are decomposed into RGB components, respectively. We set a suitable threshold to retrieve the codes from binary stripes in green and blue at each pixel. The wrapped phase map is retrieved from the sinusoidal fringes, and the codewords are retrieved from the binary stripes. We use $\hat{C}(x, y)$ to denote the retrieved codewords at pixel $(x, y)$. The fringe identification sequence at each pixel can be decoded from $\hat{C}(x, y)$. We use $\hat{q}(x, y)$ to denote the retrieved fringe identification sequence and compare it with $q_{n}$ to determine the fringe number index $k_{d}(x, y)$ at pixel $(x, y)$. Figure 2 shows the flowchart of the proposed approach.

\section{Experiment Verifications}

In order to demonstrate the proposed approach, we tested it by a color-projection-based FPP system in our laboratory. In the experimental system, the fringe patterns are generated by a HITACHI CP-X260 digital projector, and images are captured by a Duncan Tech MS3100-RGB 3-CCD digital camera with a resolution of $1392 \times 1040$ pixels. The digital camera is placed on top of the projector with a distance of $350 \mathrm{~mm}$ between their lenses. The
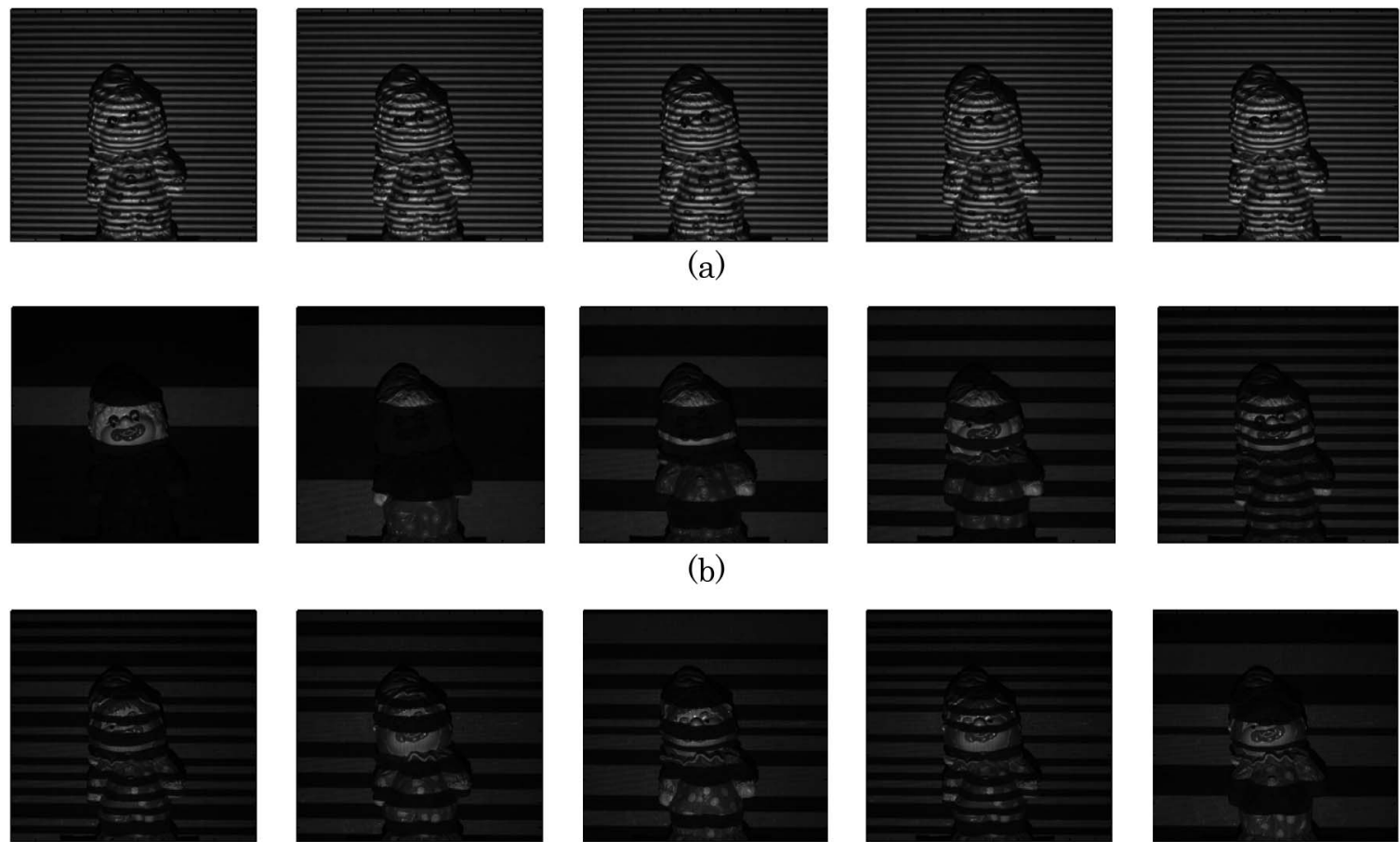

(a)
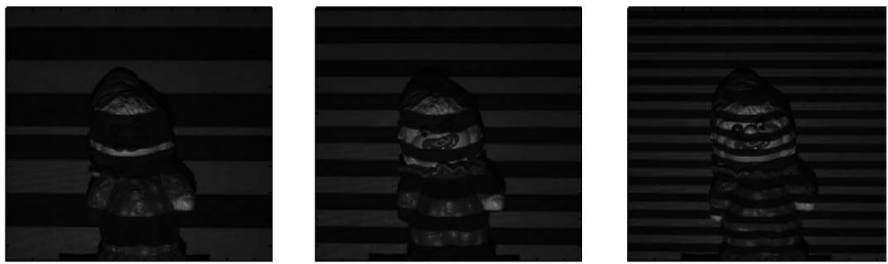

(b)
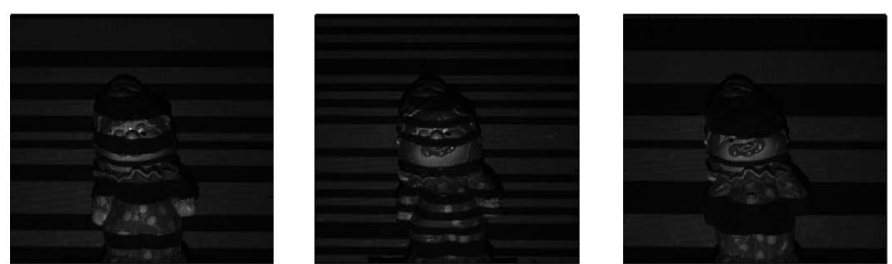

(c)

Fig. 4. (a) Captured sinusoidal fringe patterns in red channel. (b) The binary stripes in green channel. (c) The binary stripes in blue channel. 


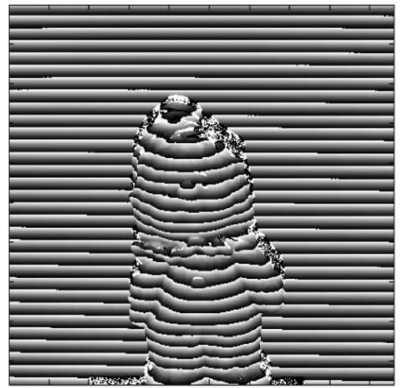

(a)

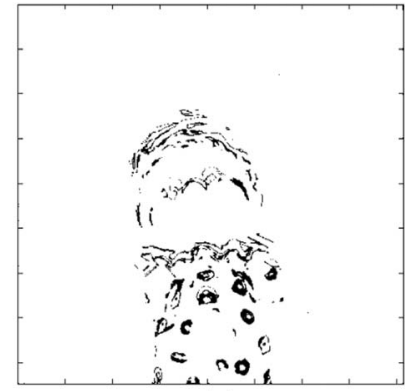

(b)

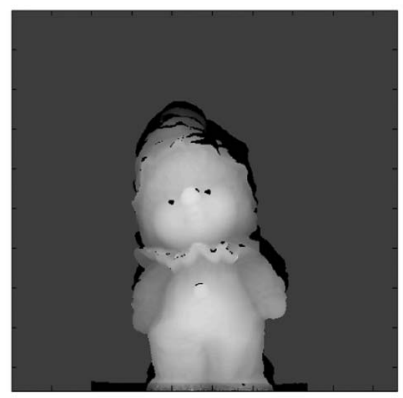

(c)

Fig. 5. (a) Wrapped phase map. (b) The pixels (denoted as black) with 1 digit error $\hat{C}(x, y)$. (c) The unwrapped phase map.

distance between the reference plane and camera lens is $895 \mathrm{~mm}$.

In our experiment, we used a color plaster doll to be the target, as shown in Fig. 3. The number of frames is five; the initial phase shift between each frame is $2 \pi / 5$. The number of fringes is 32 . The fringe identification sequences $q_{n}$ are 325 bit binary digits from 0 to 31 , where $q_{1}=[0,0,0,0,0]$ and $q_{32}=[1,1,1,1,1]$. Then $C_{n}$ is obtained by encoding each corresponding $q_{n}$ through the $(10,5)$ encoder. The figure below shows the fringe patterns projected in each frame.

As we know, when RGB color channels are used in 3D shape measurement, chromatic aberration, and cross talk between color channels are not negligible $[\underline{9}, \underline{17}, \underline{18}]$. In the proposed approach, we used the method in [9] to eliminate the color crosstalk effect. Figure 4 shows the decomposed sinusoidal fringe patterns and binary stripes images. The wrapped phase map is then obtained by taking phase detection on the sinusoidal fringe patterns. As the

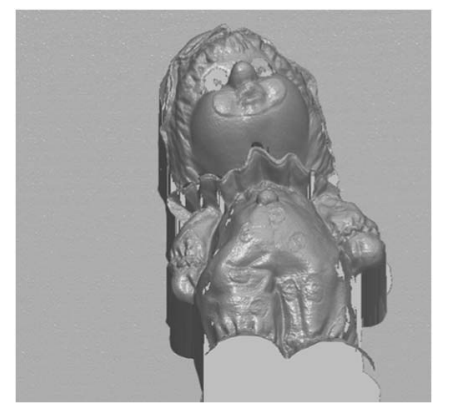

Fig. 6. 3D result obtained by the proposed algorithm.

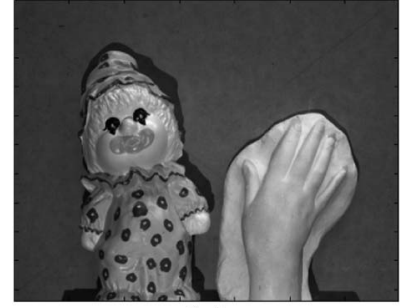

(a)

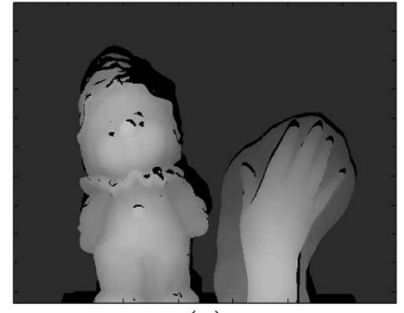

(b)
Fig. 7. (a) Appearance of targets. (b) The recovered phase map.

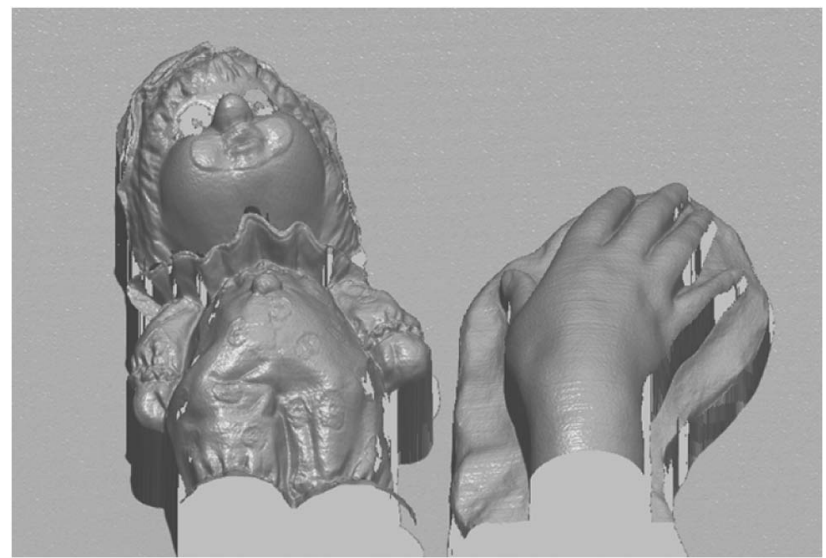

Fig. 8. 3D result obtained by the proposed algorithm.

retrieved intensity value is normalized into $255 \mathrm{lev}$ els, where 0 stands for the darkest and 255 stands for the brightest. Considering the influence of background light, we set the threshold $I_{\text {th }}=155$, which is above the middle of the range of the intensity $(0,255)$. The fringe identification sequence $\hat{q}(x, y)$ is decoded from $\hat{C}(x, y)$. In some areas, as shown in Fig. $5(\mathrm{~b})$, the retrieved $\hat{C}(x, y)$ observed 1 digit error. Such errors are then corrected when decoding $\hat{C}(x, y)$. The obtained unwrapped phase map is shown in Fig. 5(c), from which we can see that the proposed method is able to recover the phase even 1 digit error exists at $\hat{C}(x, y)$.

The 3D reconstruction result of our experiment is shown in Fig. $\underline{6}$. We also test our proposed algorithm onto the experiment of two independent objects. Figure 7(a) is a photo of the targets. Figure $7(\mathrm{~b})$ shows the recovered phase map. Figure $\underline{8}$ shows the $3 \mathrm{D}$ result.

In order to evaluate the effectiveness of the proposed method, we also calculated the rate of unsuccessfully unwrapped pixels over the total $\gamma[2,3]$. For the results presented in Figs. 5(c) and 7(c), $\gamma$ is less than $0.002 \%$, which is obviously very low in contrast to the results in [11-13].

\section{Conclusions}

A new color-projection-based 3D FPP is presented to recover the $3 \mathrm{D}$ shape of objects with large depth discontinuities and surface color. In the proposed approach, $M$ frames of sinusoidal fringe patterns 
images are used to recover the phases. Each fringe of the sinusoidal patterns was identified by a binary sequence $q_{n}$. Then we constructed a $(10,5)$ shorten extended Hamming code with a minimum Hamming distance $d_{H}$ at 4 , with which the $q_{n}$ were embedded into $2 M$ frames of binary stripes. With a colorprojection technique, the sinusoidal fringe patterns and binary stripes were projected simultaneously using RGB. The composite images were then decomposed into RGB components once captured. The wrapped phase map was obtained from the sinusoidal fringes, and the fringe identification sequences for unwrapping were obtained from the binary stripes. As been proven by the experiments, the proposed method is able to implement measurement of objects with both surface color and large depth discontinuities.

\section{References}

1. M. Takeda and K. Mutoh, "Fourier transform profilometry for automatic measurement of 3D object shapes," Appl. Opt. 22, 3977-3982 (1983).

2. S. Zhang, X. Li, and S. Yau, "Multilevel quality-guided phase unwrapping algorithm for real-time three-dimensional shape reconstruction," Appl. Opt. 46, 50-57 (2007).

3. K. Chen, J. Xi, and Y. Yu, "Quality-guided spatial phase unwrapping algorithm for fast three-dimensional measurement," Opt. Commun. 294, 139-147 (2013).

4. X. Su and W. Chen, "Reliability-guided phase unwrapping algorithm: a review," Opt. Lasers Eng. 42, 245-261 (2004).

5. S. Fang, L. Wang, P. Yang, L. Meng, and M. Komori, "Objectimage-based method to construct an unweighted quality map for phase extraction and phase unwrapping," Appl. Opt. 50, 1482-1487 (2011).

6. J. M. Huntley and H. O. Saldner, "Temporal phase unwrapping algorithm for automated interferogram analysis," Appl. Opt. 32, 3047-3052 (1993).
7. Y. Ding, J. Xi, Y. Yu, W. Cheng, S. Wang, and J. F. Chicharo, "Frequency selection in absolute phase maps recovery with two frequency projection fringes," Opt. Express 20, 13238-13251 (2012)

8. Y. Ding, J. Xi, Y. Yu, and J. F. Chicharo, "Recovering the absolute phase maps of two fringe patterns with selected frequencies," Opt. Lett. 36, 2518-2520 (2011).

9. Z. Zhang, C. E. Towers, and D. P. Towers, "Time efficient color fringe projection system for 3D shape and color using optimum 3-frequency selection," Opt. Express 14, 6444-6455 (2006).

10. P. Bao, F. Zhang, G. Pedrini, and W. Osten, "Phase retrieval using multiple illumination wavelengths," Opt. Lett. 33, 309-311 (2008).

11. Y. Wang, S. Yang, and X. Gou, "Modified Fourier transform method for 3D profile measurement without phase unwrapping," Opt. Lett. 35, 790-792 (2010).

12. W. Su, "Projected fringe profilometry using the area-encoded algorithm for spatially isolated and dynamic objects," Opt. Express 16, 2590-2596 (2008).

13. W. Su, "Color-encoded fringe projection for 3D shape measurements," Opt. Express 15, 13167-13181 (2007).

14. H. Chen, J. Zhang, and J. Fang, "Surface height retrieval based on fringe shifting of color-encoded structured light pattern," Opt. Lett. 33, 1801-1803 (2008).

15. H. J. Chen, J. Zhang, D. J. Lv, and J. Fang, "3-D shape measurement by composite patterns projection and hybrid processing," Opt. Express 15, 12318-12330 (2007).

16. W. Liu, Z. Wang, G. Mu, and Z. Fang, "Color-coded projection grating method for shape measurement with a single exposure," Appl. Opt. 39, 3504-3508 (2000).

17. Z. H. Zhang, "Review of single-shot 3D shape measurement by phase calculation-based fringe projection techniques," Opt. Lasers Eng. 50, 1097-1106 (2012).

18. Y. Hu, J. Xi, J. Chicharo, and Z. Yang, "Blind color isolation for color channel based fringe pattern profilometry using digital projection," J. Opt. Soc. Am. A 24, 2372-2382 (2007).

19. A. J. Viterbi, "Error bounds for convolutional codes and an asymptotically optimum decoding algorithm," IEEE Trans. Inf. Theory 13, 260-269 (1967).

20. T. K. Moon, Error Correction Coding, Mathematical Methods, and Algorithms (Wiley, 2005), pp. 480-522. 